\title{
Multiple Inflammatory Pseudotumors of the Liver Associated with Acute Myeloblastic Leukemia
}

\author{
Katsushi Tajima, Shigeki Kosugi, Shigemi Fuyama*, Kunihiko MaedA*, Tomohiro Hayashi, \\ Akito Yahagi, Jiro Akiba, Keijiroh Suzuki, Shinji SAtoH** and Hideo SaSaKi***
}

\begin{abstract}
A 26-year-old man, diagnosed with acute myelogenous leukemia had multiple inflammatory pseudotumors (IPT) in the liver. The patient presented complete remission after remission induction therapy, and then showed right upper quadrant discomfort and intermittent fever. An ultrasonography disclosed multiple hypoechoic nodules in the liver. A biopsy of the nodules showed focal liver cell necrosis with scant inflammatory cells, compatible with IPT. After several courses of chemotherapy, the nodules in the liver increased. The second liver biopsy of the nodule showed fibrosis. Multiple IPTs in the liver should be distinguished from abscess and metastatic nodules. (Internal Medicine 37: 542-545, 1998)
\end{abstract}

Key words: computed tomography, ultrasonography, differential diagnosis, abscess, metastasis

\section{Introduction}

An inflammatory pseudotumor (IPT) is a rare fibro-inflammatory lesion of unknown origin (1). IPT in the liver usually appears as a solitary mass. Multiple IPTs are rare $(2,3)$. Multiple IPTs are often confused with metastasis or abscess, when associated with malignant diseases and treatment with cytotoxic agents. Recently, reports of hepatic candidiasis have increased in cases of blood disorders (4). It is difficult to distinguish between multiple IPTs and hepatic candidiasis. This study presents the follow-up imaging appearance of multiple IPTs of the liver in an acute myelogenous leukemia (AML) patient. Moreover, with a repeat liver biopsy, it shows a correlation between the radiographic changes of IPT and histopathologic findings.

\section{Case Report}

A 26-year-old man developed headache and fever in May 1996. Laboratory findings are listed in Table 1 . They revealed a white blood cell count of $1,800 / \mathrm{mm}^{3}$ with $32 \%$ myeloblasts. Myelogram demonstrated the excess of myeloblasts and the patient as AML, M2 on the French-American-British classification. Chromosomal analysis of bone marrow cells revealed translocation(8;21)(q22;q22). Three positive signals were identified in $75 \%$ of bone marrow cells by fluorescent in situ hybridization. The patient received chemotherapy, according to the Japan-Adult-Leukemia-Study Group 87 protocol (BHACDMP: enocitabine, daunorubicin, 6-mercaptopurine riboside, and prednisolone). He developed remission with no sepsis after the second remission induction therapy. A repeat bone marrow examination at the termination of chemotherapy showed no leukemia cells. Before the consolidation therapy, the patient began to notice dull right upper quadrant discomfort and intermittent fever. Laboratory tests revealed an elevation of the serum C-reactive protein (CRP) level from 0.8 to $8.7 \mathrm{mg} / \mathrm{dl}$ and an increase in alkaline phosphatase (ALP) level from 56 to 138 IU (normal 39-125). The bilirubin and lactate dehydrogenase (LDH) were in the normal range.

An ultrasonography disclosed multiple hypoechoic nodules of up to $3 \mathrm{~cm}$ in the liver. A computed tomography (CT) scan showed multiple low-density masses, which were weakly isoenhanced with contrast media (Fig. 1). Magnetic resonance (MR) imaging was performed. On both T1-weighted and T2weighted images, several nodules were hyperintense relative to the liver. Other nodules demonstrated central hyperintensity on T1- and T2-weighted imagings with peripheral hypointensity on the T1-weighted image (Fig. 2).

A needle biopsy of the nodules was performed twice with ultrasonography. Histological examination showed focal liver cell necrosis with mild fibrosis and scant inflammatory cells (Fig. 3). The lesion was not encapsulated. The inflammation

From the Third Department of Internal Medicine, *the First Department of Pathology, **Division of Blood Transfusion, Yamagata University School of Medicine, Yamagata and ***the Center of Diabetes Mellitus, Nagaoka Red Cross Hospital, Nagaoka

Received for publication November 7, 1997; Accepted for publication March 16, 1998

Reprint requests should be addressed to Dr. Katsushi Tajima, the Third Department of Internal Medicine, Yamagata University School of Medicine, 2-2-2 IidaNishi, Yamagata 990-9585 
Table 1. Laboratory Findings on Admission

\begin{tabular}{lr}
\hline Hematology & \\
White blood cells & $1,800 / \mathrm{mm}^{3}$ \\
Myeloblast & $32 \%$ \\
Red blood cells & $177 \times 10^{4} / \mathrm{mm}^{3}$ \\
Hmoglobin & $6.0 \mathrm{~g} / \mathrm{dl}$ \\
Platelets & $1.2 \times 10^{4} / \mathrm{mm}^{3}$ \\
& \\
Chemistry & \\
Total protein & $7.8 \mathrm{~g} / \mathrm{dl}$ \\
Albumin & $3.6 \mathrm{~g} / \mathrm{dl}$ \\
Lactate dehydrogenase & $2,520 \mathrm{IU}$ \\
Asparate aminotransferase & $34 \mathrm{IU}$ \\
Alanine aminotransferase & $18 \mathrm{IU}$ \\
Alkaline phosphatase & $48 \mathrm{IU}$ \\
Blood urea nitrogen & $18 \mathrm{mg} / \mathrm{dl}$ \\
Creatinine & $1.1 \mathrm{mg} / \mathrm{dl}$ \\
\hline
\end{tabular}

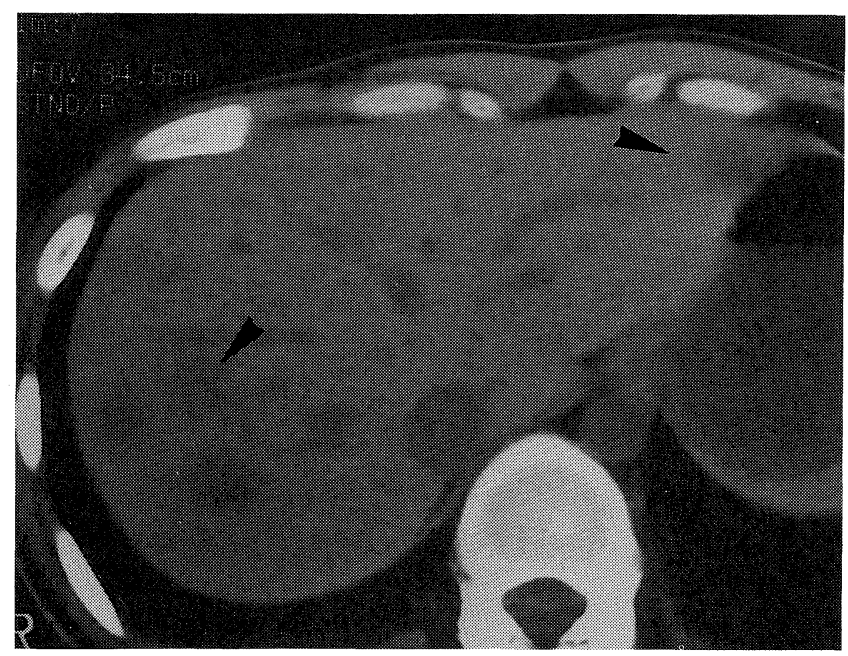

Figure 1. CT scan shows multiple ill-defined hypodense nodules (arrowheads).

consisted of lymphocytes and plasma cells. Stains for microorganisms, including Gram and Ziel-Neelsen, were all negative. Nothing was cultured. The pathological diagnosis was IPT of the liver. Within 4 weeks after the liver biopsy, his fever and right upper quadrant pain gradually resolved without any specific antibiotic therapy.

During the following months, the patient received consolidation chemotherapy, and remained asymptomatic. Follow-up $\mathrm{CT}$ scans at the termination of chemotherapy revealed an increase in the number and change in the pattern of contrast enhancement in the hepatic nodules. The delayed-phase CT scan showed an enhancement at the periphery of several nodules (Fig. 4). A second liver biopsy was again performed on the same nodule at the initial biopsy. A specimen showed concentrated fibrosis and focal liver cell necrosis surrounded by

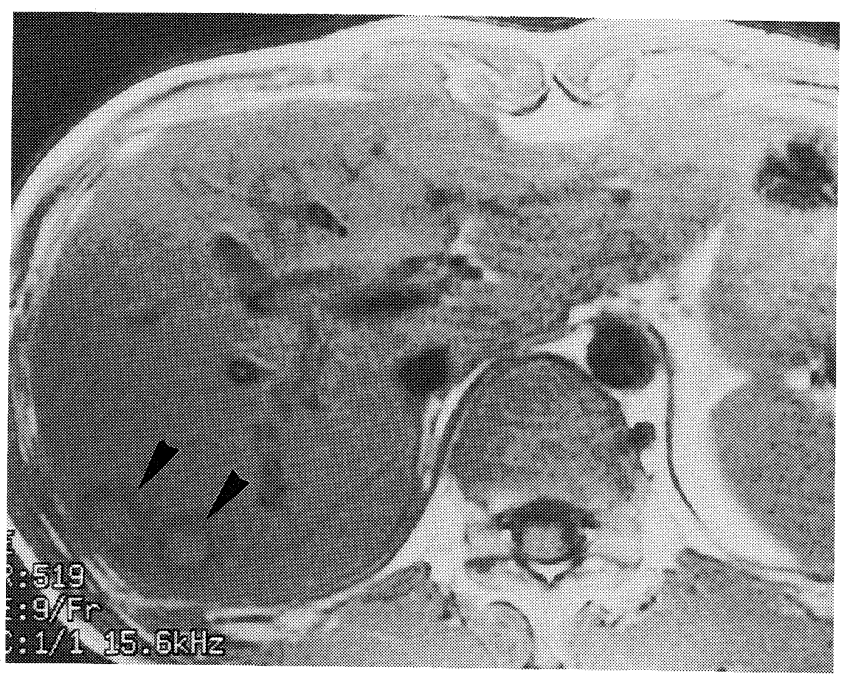

Figure 2. On a T1-weighted image, several nodules are hyperintensive with peripheral hypointensity (arrowheads).

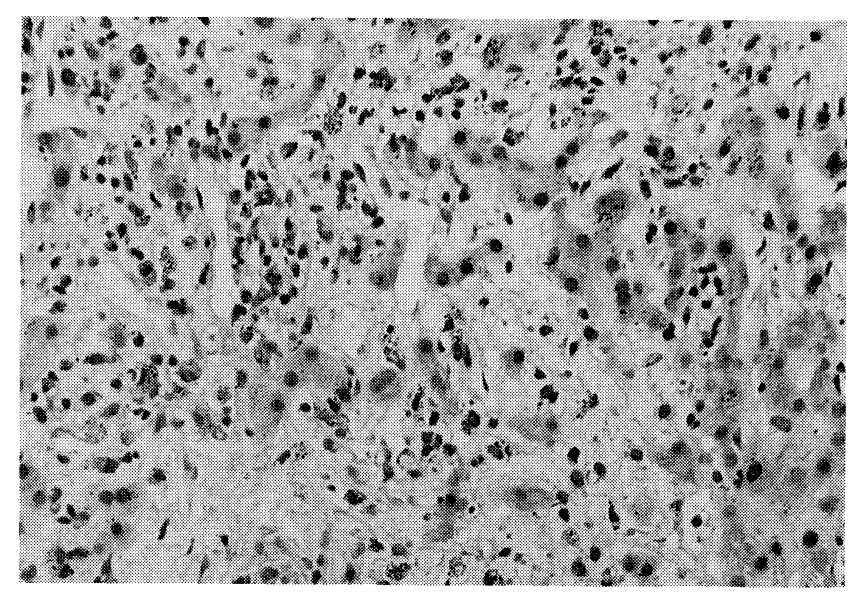

Figure 3. Initial biopsy specimen shows focal liver cell necrosis and scant inflammatory cells (HE stain, $\times 200)$.

epithelioid like cells (Fig. 5). The moderate infiltration of neutrophils, eosinophils, and lymphocytes was shown. The follow-up CT 10 months after the initial study revealed markedly smaller nodules with fine calcifications. The patient remains complete remission.

\section{Discussion}

IPT of the liver may be a rare benign process characterized by chronic inflammation rather than a single disease entity. IPT usually appears as a solitary mass, and rarely as multiple nodules $(3,5)$. Multiple IPTs should be distinguished from metastasis and abscess. In the present case, multiple nodules of the liver were initially suspected to be an abscess or metastasis because of the patient's antileukemia chemotherapy for AML. 


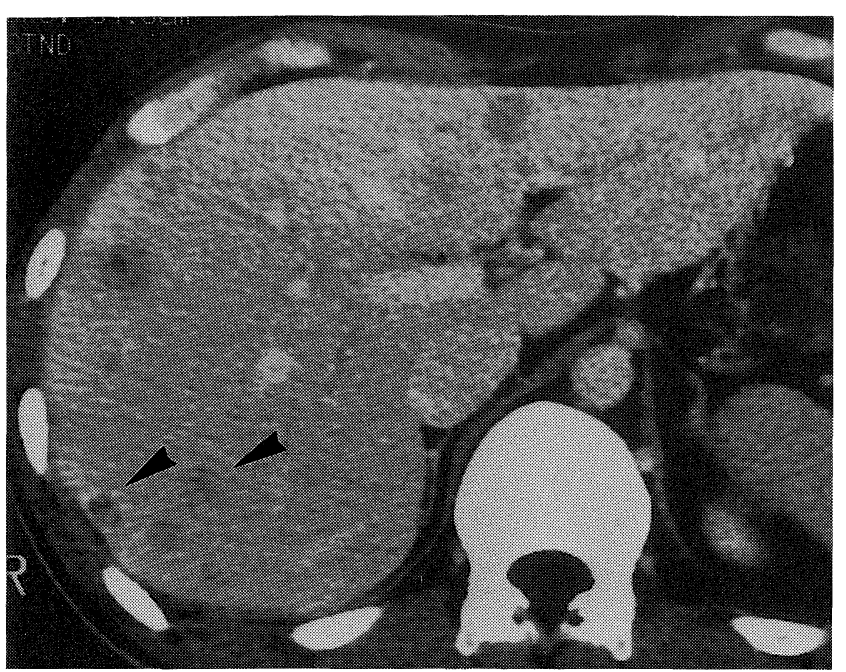

Figure 4. Delayed-phase CT scan shows an enhancement at the periphery of several nodules (arrowheads).

We performed a liver biopsy, and showed the specimen to have a characteristic appearance of IPT. Therefore, we wish to emphasize the importance of a liver biopsy in the differential diagnosis of multiple liver nodules, especially when associated with malignant diseases. Moreover, a follow-up liver biopsy may correlate dynamic changes in radiologic appearance with histopathologic findings. This case represents the first case to be reported in the literature of multiple IPTs associated with AML.

IPT is described as a hypodense lesion with no significant change after the injection of an intravenous contrast medium on CT scans (6). In our case, ultrasonography demonstrated multiple low echoic nodules, and the initial CT scan showed weak, early enhancing nodules. The follow-up CT scans demonstrated a delayed higher enhancement at the periphery in several nodules. This change in the enhancement pattern on follow-up CT scans may be attributed to the particular stage of dynamic inflammatory process. Pathologically, the initial liver biopsy specimen showed a predominantly focal liver cell necrosis with mild cellular infiltration, and the second one consisted of concentrated fibrosis along with cellular infiltration. This pathologic change may explain the delayed enhancement of IPT on follow-up CT scans. Indeed, delayed enhancement has been reported in IPT with the area of fibrosis (7).

IPT appeared homogenous or hypointense relative to the adjacent hepatic parenchyma on T1-weighted MRI, and isointense or slightly hyperintense on T2-weighted MRI (6). In our case, several nodules demonstrated central enhancement on T1- and T2-weighted imagings with peripheral hypointensity on T1-weighted MRI. These MR imagings may show a central hemorrhage in several nodules.

The differential diagnosis should encompass liver abscess, metastasis, and hepatocellular carcinoma. A liver abscess is an inflammatory liver disease, with symptoms and laboratory data similar to those of IPT. A typical liver abscess is characterized by a liquefactive necrosis of neutrophils, plasma cells, and

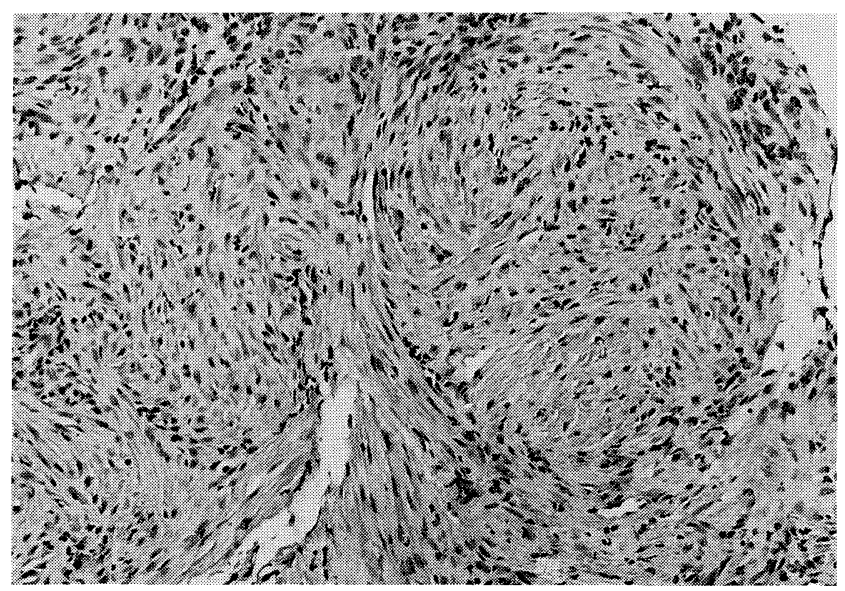

Figure 5. Repeat biopsy specimen shows fibrous tissue with inflammatory cells $($ HE stain, $\times 100)$.

histiocytes. Therefore, CT scans of a typical liver abscess show central water density. However, an atypical liver abscess with incomplete liquefaction may mimic IPT on CT scans, and thus may be difficult to differentiate from IPT. Metastatic tumors with fibrosis can show delayed enhancement on a CT scan. On the basis of CT findings alone, hepatic metastasis may also be difficult to distinguish from IPT. In our case, multiple low echoic nodules were found, and suspected to be metastasis or multiple liver abscess. However, these nodules were not compatible with CT findings of a typical liver abscess or metastatic nodules. Radiologic findings of IPT have been known to be nonspecific, and combined judgment is required.

The pathogenesis of IPT remains unclear. In some cases, IPT has had possible antecedent factors, including trauma and infections (8). IPT may reflect a nonspecific inflammatory process of these factors. In our case, IPT appeared when the patient entered complete remission and recovered after the second remission induction chemotherapy. Several courses of antileukemia chemotherapy increased the number of hepatic nodules. We identified histologically no organisms in the biopsy specimen, and nothing was cultured. The antibiotic therapy evidenced no changes in the hepatic nodules. These results may suggest no direct linkage of microorganisms to IPT in this patient. The initial biopsy specimen showed small focal liver cell necrosis with no prominent inflammatory cells, a histological feature which may mimic drug-induced hepatitis. In our case, IPT may be attributable to the chemotherapy, which could trigger and affect the immunological responses of the host. The recognition of this form of IPT with AML is important for clinicians and radiologists to avoid inappropriate antileukemia and antibiotic chemotherapy.

\section{References}

1) Nam KJ, Kang HK, Lim JH. Inflammatory pseudotumor of the liver: CT and sonographic findings. Am J Roentgenol 167: 485, 1996.

2) Isobe $\mathrm{H}$, Nishi $\mathrm{Y}$, Fukutomi $\mathrm{T}$, et al. Inflammatory pseudotumor associated with acute myelomonocytic leukemia. Am J Gastroenterol 86: 238, 


\section{Inflammatory Pseudotumor}

1991.

3) Horiuchi R, Uchida K, Kojima T, Shikata T. Inflammatory pseudotumor of the liver: clinicopathological study and review of the literature. Cancer 65: $1583,1990$.

4) Blade J, Lopez-Guillermo A, Rozman C, et al. Chronic systemic candidiasis in acute leukemia. Ann Hematol 64: 240, 1992.

5) Li GH, Li JQ, Lin YZ. Inflammatory pseudotumor of the liver. J Surg Oncol 42: 244, 1989.
6) Abehsera M, Vilgrain V, Belghiti J, Flejou JF, Nahum H. Inflammatory pseudotumor of the liver: radiologic-pathologic correlation. J Comput Assist Tomogr 19: 80, 1995.

7) Itai Y, Ohtomo K, Kokubu T, et al. CT of hepatic masses: significance of prolonged and delayed enhancement. Am J Roentgenol 146: 397, 1986.

8) Vujanic GM, Milovanovic D, Aleksandrovic S. Aggressive inflammatory pseudotumor of the abdomen 9 years after therapy for Wilms tumor: a complication, coincidence, or association. Cancer 70: 2362, 1992. 